\title{
ASPECTOS BIOLÓGICOS Y ECOLÓGICOS DEL OSO DE ANTEOJOS (Tremarctos ornatus, Ursidae) EN LA ZONA ANDINA DE ECUADOR Y PERSPECTIVAS PARA SU CONSERVACIÓN BAJO EL ENFOQUE DE ESPECIES PAISAJE
}

\author{
BIOLOGICAL AND ECOLOGICAL ASPECTS OF THE SPECTACLED BEAR \\ (Tremarctos ornatus, Ursidae) IN THE ECUADOREAN ANDEAN ZONE AND \\ CONSERVATION PERSPECTIVES UNDER THE LANDSCAPE SPECIES APPROACH
}

\author{
Pablo Sandoval Guillén*1 [i] y Patricio Yánez Moretta² ${ }^{2}$
}

1 Ingeniería en Biodiversidad y Recursos Genéticos, Universidad Tecnológica Indoamérica, Av. Machala y Sabanilla, Quito, Ecuador.

${ }^{2}$ Carrera de Gestión Ambiental y Turismo, Universidad Internacional del Ecuador, Av. Simón Bolívar y Jorge Fernández, Quito, Ecuador.

*Autor para correspondencia: apyanez@hotmail.com

\begin{abstract}
Resumen
El oso andino u oso de anteojos (Tremarctos ornatus) ha habitado Sudamérica por más de cinco millones de años, y es el único representante viviente de los osos de nariz corta, un grupo que habitó solamente en el continente americano. Dentro de Ecuador es considerado en peligro de extinción, principalmente debido a la fragmentación y pérdida de hábitats naturales en la región andina, causada por actividades antrópicas productivas (ganadería y agricultura) y de extracción de recursos naturales. Este oso es un mamífero que necesita de grandes extensiones para poder alimentarse y buscar pareja. El oso andino es importante para las zonas que habita, principalmente el bosque andino y el páramo, debido a su eficiente rol como dispersor de semillas. En el presente trabajo se analizan aspectos de esta especie en Ecuador, utilizando el enfoque teórico de 'especies paisaje' para proponer actividades en pro de la conservación de este mamífero y de los hábitats en los que se desarrolla. Este enfoque permite evaluar de forma sistemática la calidad del paisaje en términos de los requerimientos biológicos de una especie de interés (en este caso, Tremarctos ornatus) y de los usos humanos del paisaje; de igual forma considera características del área de conservación como su variación. Palabras clave: Conservación biológica, Tremarctos ornatus, Andes ecuatorianos, especies.
\end{abstract}


The Andean bear (Tremarctos ornatus) has inhabited South America for more than five million years, and he is the only living representative of the short-nosed bears, a group that only lived on the American continent. In Ecuador he is considered an endangered species, mainly because the loss of natural habitats in the Andes, due to the pressure of productive anthropic activities (mainly, livestock and agriculture) and extraction of natural resources. This bear is a mammal that needs large areas to eat and find a mate. The Andean bear is important for cloud forests and paramos due to his efficient role as seed scatter. In this work, the 'landscape species' theoretical approach is applied in order to propose activities to conserve the Andean bear and the places that inhabits. This approach also allows to evaluate in a systematic way the quality of the landscape in terms of biological requirements of the species (here, Tremarctos ornatus) and the landscape human uses; likewise, it also considers the size and limits of the conservation area and its internal variation.

Keywords: Biological conservation, Tremarctos ornatus, Ecuadorian Andes, Landscape Species.

Forma sugerida de citar: Sandoval-Guillén, P. y Yanéz-Moretta, P. (2019). Aspectos biológicos y ecológicos del oso de anteojos (Tremarctos ornatus, Ursidae) en la zona andina de Ecuador y perspectivas para su conservación bajo el enfoque de especies paisaje. La Granja: Revista de Ciencias de la Vida. Vol. 30(2):19-27. http://doi.org/10.17163/lgr.n30.2019.02.

IDs Orcid:

Pablo Sandoval Guillén: https: / orcid.org/0000-0001-8157-2876

Patricio Yánez Moretta: https:/ /orcid.org/0000-0003-4436-7632 


\section{Introducción}

Varias regiones en Latinoamérica han sido propuestas como 'hotspots' (áreas con especial concentración de diversidad biológica), 'threatspots' (áreas con tal diversidad, pero con amenazas claras hacia ella) o como 'países con números altos de especies amenazadas' (Cole, Reeder y Wilson, 1994; Ceballos y Brown, 1995; IUCN, 1996). Dentro de este contexto, cabe mencionar que los mamíferos y las aves, como grupos mejor conocidos y más estudiados, suelen ser considerados como los organismos animales generalmente emblemáticos, en torno a los cuales suelen girar actividades de planificación y ejecución de la conservación de hábitats y ecosistemas naturales.

La riqueza de especies, valores de biodiversidad y rangos de endemismo de mamíferos para cada país han sido utilizados de manera conjunta como medidas de caracterización de la pérdida de hábitat y crecimiento poblacional humano, así como para identificar las amenazas antrópicas globales sobre la diversidad biológica (Sisk y col., 1994). A pesar de ello, todavía existen algunos vacíos en el conocimiento de este grupo de vertebrados en regiones neotropicales. Entre los mamíferos emblemáticos de zonas andinas, el oso andino (Tremarctos ornatus) se encuentra catalogado como especie vulnerable (VU) dentro de la Lista Roja de la Unión Internacional para la Conservación de la Naturaleza (IUCN, 2018) y en peligro de extinción en Ecuador (Cuesta, Peralvo y Sánchez, 2001).

Uno de los problemas más desafiantes en la conservación de biodiversidad en la zona andina de Ecuador es proteger especies grandes, como el oso de anteojos (Castellanos, 2010) o el tapir de montaña (Álvarez y Yánez, 2017), y al mismo tiempo satisfacer las necesidades de las personas que comparten espacios con ellas. Los grandes carnívoros, por ejemplo, son particularmente difíciles de conservar porque compiten directa o indirectamente con los humanos por el espacio y los recursos (Treves y col., 2006); los carnívoros tienen efectos directos sobre la abun- dancia de herbívoros, carroñeros y efectos indirectos sobre la vegetación y las redes alimenticias a través de cascadas tróficas (McFarland, 2009), lo que hace que su conservación sea importante y compleja.

La conservación del oso de anteojos en los países andinos, en donde las áreas protegidas y los hábitats de vida silvestre se encuentran incrustados en una matriz de usos heterogéneos, generalmente con cambios rápidos en el uso y/o tenencia de la tierra, es particularmente difícil (Peyton y col., 1998; Ferraro, 2002; Himley, 2009).

\subsection{La región andina de Ecuador y países vecinos como hábitat natural del oso de anteojos}

Clásicamente suele considerarse al hábitat como todos los recursos y características de un lugar, que permiten la presencia de un organismo (Begon, Harper y Townsend, 1999); por lo tanto, un animal puede usar un hábitat de diferentes formas, de acuerdo al acceso y la disponibilidad de éste, así como de sus requerimientos particulares (Cuesta, Peralvo y Sánchez, 2001). La disponibilidad y acceso a tal hábitat pueden variar a lo largo del tiempo en función de factores ambientales como el clima y factores biológicos de la especie de interés, así como de las comunidades que estructuran el sistema local (Begon, Harper y Townsend, 1999).

A nivel de Sudamérica, el oso de anteojos habita en ambientes de bosques nublados y páramos de seis países: Colombia, Venezuela, Ecuador, Perú, Bolivia y noroccidente de Argentina (Castellanos, 2010). La presencia de esta especie en su zona más austral, el noroccidente de Argentina, se fundamenta en el encuentro de fragmentos de ADN, extraídos de pelo y heces (Teta y col., 2018). En Ecuador se reporta su presencia principalmente en bosques nublados y páramos andinos, generalmente dentro de áreas protegidas, pero eventualmente también fuera de ellas (Cuesta, 2000; Goldstein y Cancino, 2001; PUCEq, 2018) (Figura 1). 


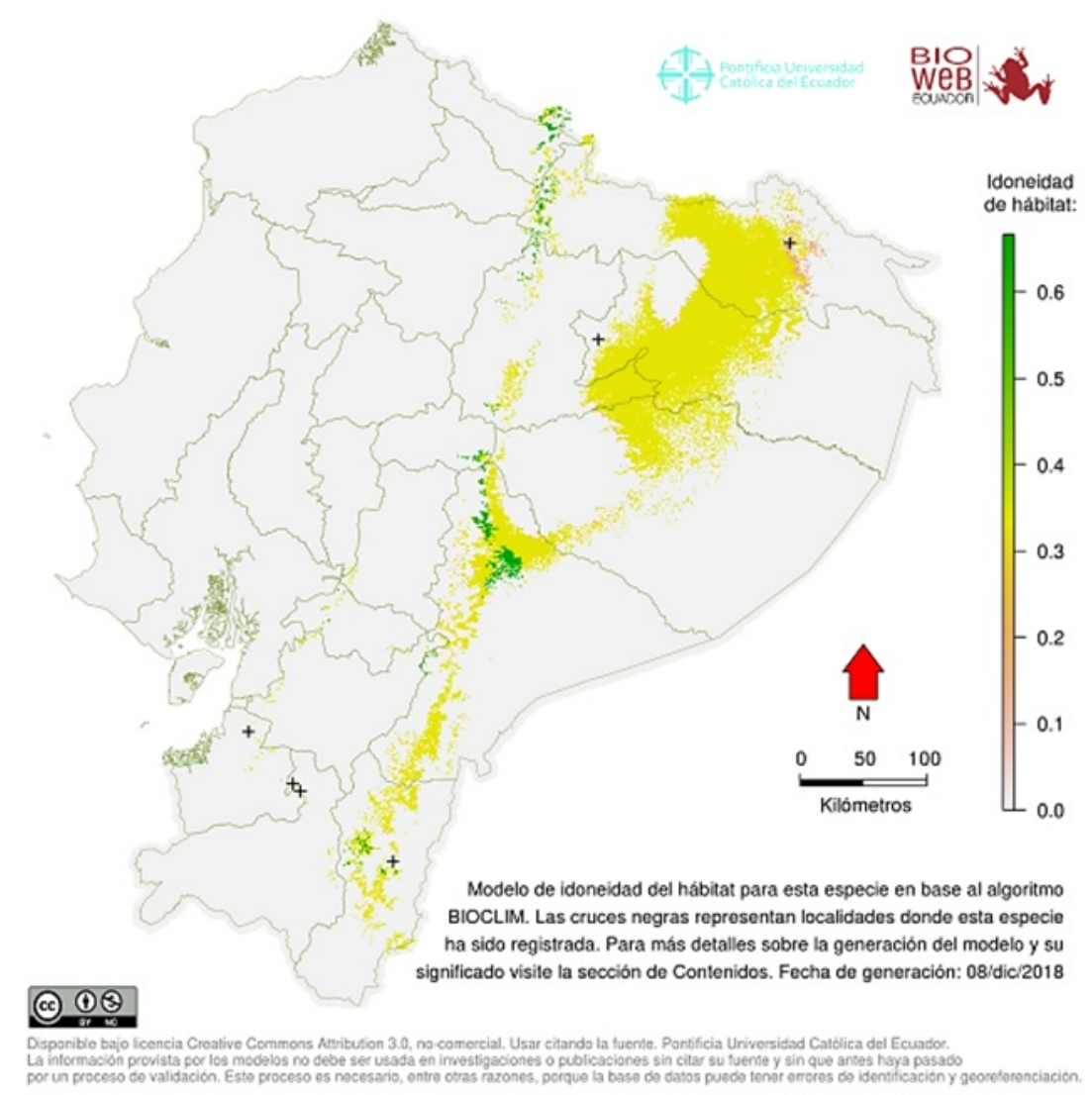

Figura 1. Mapa de distribución potencial del oso de anteojos.

Fuente: PUCE-q, (2018).

Algunos registros de su presencia incluyen bosque nublado y páramo a regiones contiguas de bosque subtropical. Su presencia en estos tres ecosistemas se relaciona con registros efectuados en las provincias de Pastaza, Morona Santiago, Zamora Chinchipe, Sucumbíos, Napo, Chimborazo, Cañar, Azuay, Loja, Carchi, Imbabura, Pichincha, Cotopaxi, Bolívar, Tungurahua y El Oro (Tirira, 2007). Atendiendo a una nomenclatura más estandarizada de las regiones naturales, se puede mencionar que se encuentra en: Bosque Pie montano Occidental, Bosque Montano Occidental, Páramo, Bosque Montano Oriental, Bosque Pie montano Oriental (Castellanos y Boada, 2018).

\subsection{Descripción de la especie}

El oso de anteojos, también conocido como oso andino, oso achupallero, oso frontino $\mathrm{u}$ oso anteojudo, es una especie única de los Andes. Es grande y de cuerpo robusto, los machos miden entre 1,8-2,0 m de altura, pesan hasta $175 \mathrm{~kg}$, las hembras son algo más pequeñas (Tirira, 2007). Los machos y hembras adultos tienen un cuello musculoso, corto, orejas cortas y hocico marrón oscuro, su pelaje negro es grueso y abundante. Presentan además una combinación de manchas blancas o café claras alrededor de los ojos, que pueden extenderse hasta la quijada, garganta y pecho (Figuras 2 y 3); el tamaño, coloración y forma de estas manchas suelen ser diferentes en cada individuo y son características que suelen ser usadas para su identificación a este nivel (Castellanos, 2010; Tirira, 2007).

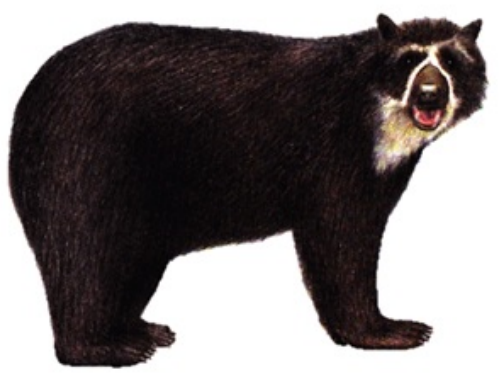

Figura 2. Apariencia de un oso de anteojos adulto. Fuente:Tirira, (2007). 


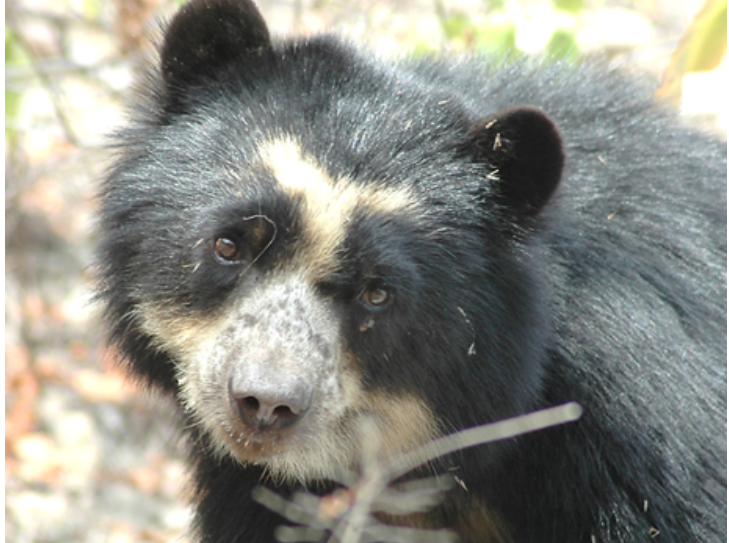

Figura 3. Oso de anteojos (Tremarctos ornatus). Fuente:Appleton, (2017)

Es un mamífero de hábitos diurnos y nocturnos, terrestre, parcialmente arborícola y solitario; también es un animal omnívoro (come frutos, materia vegetal y carne, llegando incluso a alimentarse de carroña de ser necesario). Generalmente, su dieta incluye frutos maduros, cogollos de bromelias, partes tiernas de palmas, bulbos de orquídeas o incluso cortezas de árboles (Castellanos, 2005). Sin embargo, sus hábitos alimenticios pueden cambiar acorde al lugar en el que se encuentre y de la disponibilidad de los recursos (Peralvo, Cuesta y Manen, 2005), y si el alimento es escaso, puede incluso llegar a cazar venados, roedores y en ocasiones terneros (Goldstein, 2002; Castellanos, 2005). Suele construir nidos tanto en el suelo como en los árboles altos con el propósito de descansar y para alimentarse sin interrupciones (Goldstein, 2002).

\subsection{Objetivo y aspectos metodológicos}

El presente documento busca compartir con la sociedad académica nacional y regional información de interés para el conocimiento y la conservación de una especie emblemática del oso de anteojos. Se fundamentó en una revisión crítica de literatura específica (considerando publicaciones técnicas y/o científicas producidas principalmente en las últimas dos décadas en torno a esta especie de mamífero y sus hábitats), así como la generación de opiniones de los autores sobre el rol ecológico y de conservación, presente y futuro de esta especie en sus hábitats naturales en Ecuador.

\section{Biología reproductiva}

Gran parte del comportamiento de apareamiento de esta especie permanece sin estudiar. Los machos y las hembras se juntan para aparearse entre los meses de abril y junio. La pareja permanece junta durante 1 a 2 semanas, copulando varias veces durante este período (International As- sociation for Bear Research and Management, 1999).

Estudios de genética molecular realizados por RuizGarcía, (2003) en poblaciones de osos de cinco países andinos muestran una alarmante realidad: la variabilidad genética del oso andino es relativamente baja. Las poblaciones del Ecuador poseen la menor variabilidad, posiblemente debido a que la fragmentación de sus hábitats es mayor en Ecuador, lo cual genera poblaciones de osos más aisladas y con mayor riesgo de desaparecer (Kattan y col., 2004).

\section{Importancia ecológica en el turis- mo cultural y de naturaleza}

El oso de anteojos es el único representante viviente en Sudamérica de toda la familia Ursidae, lo cual le da un derecho particular de existir y a la comunidad académica una gran obligación ética para facilitar su conservación in situ. El ser humano, como el principal agente transformador del ambiente, tiene la obligación y responsabilidad de conservar no solo al oso andino sino también a las especies silvestres con las que comparte hábitats.

Sin embargo, y a pesar de esta necesidad ética, poco se conoce aún sobre la ecología de este oso en Ecuador y su rol en los ecosistemas que habita. Estudios en Bolivia han demostrado que es un activo agente dispersor de semillas y que el paso de éstas por su tracto digestivo no afecta su viabilidad (Rivadeneira, 2008).

Por tanto, este mamífero resulta muy importante para las zonas que habita, principalmente para algunas especies vegetales de bosque andino y páramo, debido a su rol efectivo de dispersor al depositar semillas con mayor velocidad de germinación en zonas aptas. Por otra parte, en la mayor parte de las veces el oso de anteojos tumba troncos completos para alcanzar sus frutos y alimentarse (Troya, 2002); de esta manera, dentro del bosque se abren espacios por donde entra más luz solar y el agua de lluvia llega directamente al suelo. Así, el oso ayuda en parte a la renovación de la vegetación del bosque, ya que muchas plantas necesitan tener un espacio abierto por encima de ellas (claros) para crecer mejor. Sus excrementos también contribuyen en alguna medida a abonar el suelo del bosque. Además, sirven de alimento a muchos pequeños organismos como escarabajos estercoleros, pequeños cucarrones, larvas de insectos, mariposas (Castellanos, 2003).

Es un gran escalador y cuando sube a los árboles para alimentarse o dormir construye plataformas doblando y partiendo algunas ramas. Muchas de estas ramas o palos que estaban acumulados en el dosel del bosque caen al suelo para descomponerse y convertirse en abono orgánico. De esta manera, el oso ayuda a evitar que se acumule 
demasiada materia orgánica en la parte alta de los árboles y genera huecos en el dosel o subdosel por donde llega la luz del sol directamente al suelo del bosque (Suárez, 1985).

El oso andino también ha sido un animal emblemático, involucrado en el desarrollo de muchas de nuestras culturas indígenas y campesinas en los países andinos. Para muchos es sagrado, para otros el hermano mayor del ser humano. En las tradiciones orales de los pueblos se encuentra al oso en leyendas, cuentos, canciones y mitos (Lameda y Del Moral, 2008).

El oso andino aparece reflejado en los nombres comunes de algunas plantas, como 'la mano de oso' (Oreopanax bogotensis) y 'la hierba del oso' (Xerophyllum tenax). También es muy común encontrar sitios o lugares que reciben sus nombres honrando al oso andino: el Alto del Oso (Colombia), la Cueva del Oso (Ecuador), Quebrada El Oso (Colombia, Perú, Venezuela), Vereda del Oso (Colombia), entre otros. De esta manera, el oso andino hace parte del patrimonio cultural y de la cosmovisión de los países de la región andina.

\section{Aspectos en torno al cuidado y conservación actuales del oso an- dino bajo el enfoque de especies paisaje}

\subsection{El modelo de conservación basado en especies paisaje}

El conflicto oso de anteojos - ganado es un elemento digno de ser analizado y abordado bajo el esquema conceptual para planificación de conservación de especies paisaje (Sanderson y col., 2002), debido a las características particulares del conflicto, que incluyen componentes ecológicos, sociales y económicos. Una planificación efectiva en conservación o en manejo de conflictos debe definir de manera clara los elementos que son biológica, económica y socialmente relevantes para la especie o el conflicto en cuestión, de manera que se pueda planificar la resolución de los mismos a una escala adecuada (Poiani y col., 1998; Whited y col., 2000), preferiblemente con un enfoque ecosistémico y/o de ecología de paisajes.

El enfoque de especies paisaje concentra sus esfuerzos en un modelo explícito espacialmente que evalúa sistemáticamente el paisaje en términos de los requerimientos biológicos de una especie de interés (en este caso, Tremarctos ornatus) y de los usos humanos que se den a tal paisaje. Este enfoque utiliza los requerimientos de la especie para definir los límites del área de conservación (la extensión) y la variación dentro de ella (el grano) (Sanderson y col.,
2002). El modelo conceptual de conservación basado en especies paisaje considera primordialmente la identificación de:

1. El paisaje biológico de la especie.

2. El paisaje de las diferentes actividades humanas.

3. Espacios y tiempos en los que las actividades humanas podrían poner en peligro a las poblaciones de la especie paisaje.

4. Un paisaje focal potencial para la conservación de la especie, basado en los elementos locales necesarios y las intervenciones diferentes requeridas para la conservación de la población biológica escogida (Sanderson y col., 2002).

\subsection{La escogencia de la especie}

Teóricamente, cualquier especie podría ser considerada como candidata a especie paisaje, pero en la práctica, considerar muchas especies como candidatas haría excesivamente largo y oneroso el proceso de selección. En vista de ello, organizaciones como la WCS sugieren que el grupo inicial de especies candidatas a especie paisaje para una zona determinada consiste en pocas especies con una probabilidad razonable de ser seleccionadas y que cumplan con uno o más de los cinco criterios siguientes (WCS: Wildlife Conservation Society, 2002):

- Área: la especie candidata a especie paisaje deberá distribuirse ampliamente en hábitats naturales, de tal manera que si se protege su zona de vida se protege a la vez a muchas otras especies de flora y fauna al mismo tiempo, lo que se conoce como Efecto Sombrilla (Umbrella species) (WCS: Wildlife Conservation Society, 2002). En este sentido el oso de anteojos, por lo que se ha mencionado anteriormente y por su distribución en hábitats naturales andinos de Colombia, Venezuela, Ecuador, Perú y norte de Argentina, es un fuerte candidato para especie paisaje.

- Heterogeneidad: algunas especies requieren más que simplemente áreas grandes. En muchos casos, una especie silvestre candidata a especie paisaje necesita una variedad de hábitats naturales disponibles y tipos de vegetación diferentes para su dispersión, forraje, reproducción y supervivencia en épocas favorables y desfavorables (WCS: Wildlife Conservation Society, 2002). Nuevamente, el oso andino reúne estas condiciones.

- Vulnerabilidad: otra consideración en la selección de una especie paisaje es el número y severidad de las amenazas que la afectan. Las amenazas pueden ser clasificadas de acuerdo a la probabilidad de su ocurrencia, la rapidez con la que ocurren o pueden ocurrir, su severidad y el área que pueden afectar, 
en relación a la especie candidata (WCS: Wildlife Conservation Society, 2002). El oso de anteojos en la actualidad está catalogado como especie vulnerable por la IUCN, (2018), por lo que su protección y de los hábitats que ocupa es necesaria y urgente.

- Funcionalidad ecológica: algunas especies tienen efectos particularmente fuertes en la estructura y función de los ecosistemas naturales: por ejemplo, los castores originan zonas pantanosas al construir diques en los ríos (WCS,2002); los tapires y osos de anteojos distribuyen semillas en los sistemas que visitan y los depredadores mayores pueden controlar la abundancia y composición de las comunidades de presas. Dados estos efectos importantes sobre otras especies, el mantenimiento de poblaciones saludables de las especies paisaje y de sus hábitats, como el caso del oso andino, ayudará a conservar de manera saludable a los ecosistemas en los que se desarrolla.

- Importancia socioeconómica: el último criterio para la selección de una especie paisaje es su importancia socioeconómica. Algunos ejemplos demuestran que el ambiente social dentro y en los alrededores de las áreas protegidas puede afectar drásticamente los resultados de la conservación. Dado que las especies paisaje recorren grandes extensiones y encuentran en este accionar una amplia variedad de hábitats y de tipos de uso de tierras, es muy probable que tengan contacto con gente y sus actividades (WCS: Wildlife Conservation Society, 2002). Esto precisamente es lo que sucede con el oso de anteojos, en un sentido negativo para el ser humano puede afectar cosechas, molestar al ganado, competir por el espacio o por los recursos; en un sentido positivo este oso tiene importantes beneficios como el ser un potente ícono cultural en la ruralidad andina y proveer oportunidades para la generación de ingresos a las comunidades humanas locales a través del desarrollo de actividades de ecoturismo, como se ha descrito para otra especie importante como el tapir andino en estudios similares (Álvarez y col., 2017).

\subsection{Programas y planes en pro de la conser- vación del oso de anteojos como especie emblemática en Ecuador}

La Fundación Natura y la Fundación EcoCiencia en Ecuador han trabajado en las últimas décadas (1980-2010, principalmente) con el gobierno nacional apoyando a la gestión del manejo de tres áreas protegidas en los que se encuentra el oso de anteojos (Podocarpus, CotacachiCayapas, Cayambe-Coca). Por otro lado, el programa de conservación del oso Andino en el Noroccidente del Distrito Metropolitano de Quito utiliza al oso como especie paraguas para la conservación de la salud ambiental de esta región (Secretaría de Ambiente, 2014). Las acciones que se implementan para apoyar la conservación del oso apoyan también directa o indirectamente a la conservación y recuperación de los remanentes de vegetación naturales. Estos esfuerzos de conservación sin duda alguna deben ser ampliados y apoyados por las instituciones de gestión e investigación que fueren pertinentes.

\section{Conclusiones y recomendaciones}

Las poblaciones de oso andino desde la época prehispánica han sido objeto de mucha presión por parte del ser humano. En Ecuador se los cazaba por temor, por deporte, por obtener productos de su cuerpo o porque eventualmente eran considerados plaga para cultivos y/o animales de granjas.

Actualmente, las amenazas principales que enfrenta son la caza furtiva y la destrucción de su hábitat natural, lo que hace que las pocas poblaciones que aún quedan en Ecuador se encuentren generalmente aisladas entre sí. A estos dos factores se le suma el hecho de que el hábitat principal del oso andino se encuentra en una de las zonas de mayor crecimiento y desarrollo económico y social en Ecuador: las provincias andinas.

Consideramos que algunas medidas para colaborar con el mantenimiento de esta especie en sus hábitats naturales, además de que se la considere oficialmente como Especie Paisaje para Ecuador y posiblemente también para los otros países andinos donde se encuentre, comprenden:

Evitar la cacería del oso con las medidas y acciones pertinentes; actualmente, la ley prohíbe su cacería y comercialización; sin embargo, ameritaría algo más de control para que llegue a cumplirse plenamente la norma. Cabe recalcar que estos animales son tímidos y asustadizos; tratan de evitar la presencia del ser humano y no constituyen amenaza alguna para nosotros. Si en una zona hay problemas con un oso andino se debe informar a la entidad ambiental local competente; ésta indicará que hacer. La cacería no es la solución y con esta práctica solo se contribuye a aumentar el riesgo de extinción de esta especie.

No talar el bosque andino (el nublado ni el de pie de monte) ni el subtropical, así como no modificar los páramos. Además de ser el hogar del oso andino, estos ambientes sirven para la protección de cuencas y aseguran el abastecimiento de agua en la región para el beneficio de la gente. Por otra parte, el bosque y el páramo son el hogar de muchas otras especies de animales y plantas beneficiosas para el ecosistema y para el ser humano, algunas de ellas además no se encuentran en ninguna otra región del planeta. De esta manera, si se protege el hogar 
del oso andino también se preserva el hogar de varias plantas y animales que comparten su hábitat. Resultan indispensables las campañas de recuperación y protección de los bosques andinos y páramos. El deterioro de estos sistemas implica la pérdida de fuentes de agua, indispensables para cultivos o consumo humano. Se debe procurar reforestar las áreas donde antes existía bosque andino con especies nativas, pues los que aún quedan se encuentran de manera discontinua y fragmentada. Se pueden desarrollar planes de restauración de bosques que conecten esos fragmentos y así se ayudaría a que el oso andino tenga un área más amplia por la cual desplazarse.

Los gobiernos (nacional, provinciales, municipales) deben tomar acciones eficaces de control al tráfico ilegal de especímenes de oso de anteojos o partes de su cuerpo. También se debe pensar en aplicar estímulos para aquellos que promuevan de alguna manera la protección de esta especie y/o de los hábitats en los que se encuentra o de los que depende directamente. Las organizaciones no gubernamentales interesadas en la conservación de esta especie deben tener la capacidad, facilitada por el Gobierno Nacional, de canalizar recursos nacionales e internacionales para la adquisición y/o administración de tierras que aseguren la conservación de los hábitats naturales del oso andino. Las universidades y centros de investigación, tanto privados como públicos, que efectúen actividades y programas en ciencias biológicas y ambientales deben estar en capacidad de ofrecer asesoría técnica y científica a las comunidades rurales y entidades gubernamentales y no gubernamentales respecto a los criterios que se deben tener en cuenta para la conservación y recuperación de las poblaciones de oso andino y los hábitats que ocupa.

\section{Agradecimientos}

A la Dra. Mónica Paéz (UTI), por los comentarios y sugerencias de mejora a las versiones iniciales del manuscrito.

\section{Referencias}

Álvarez, P. y P. Yánez (2017). «Actividades de conservación y de educación ambiental en torno a una especie emblemática, el tapir de montaña (Tapirus pinchaque), en sectores andinos del sur de Ecuador.» En: INNOVA Research Journal 2.8, 1-9. Online: https: / / bit.ly/ 2N0tr5h.

Álvarez, P. y col. (2017). «El Tapir de montaña (Tapirus pinchaque), como especie bandera en los Andes del sur del Ecuador.» En: INNOVA Research Journal 2.8, 86-103. Online: https://bit.ly/2ZnuZN7.

Appleton, R. (2017). Spectacled Bear. Tremarctos ornatus.
Begon, M., J. Harper y C. Townsend, eds. (1999). Ecología. Online: https:/ / bit.ly/2ZdR4yx: Omega.

Castellanos, A. (2003). «Datos ecológicos del oso andino Tremarctos ornatus en la reserva Alto Chocó, Ecuador». En: Memorias de las XXVII Jornadas Ecuatorianas de Biología. Ed. por Universidad Central del Ecuador. Quito-Ecuador.

- (2010). Guía para la rehabilitación, liberación y seguimiento de osos andinos. Imprenta Anyma. Quito.

Castellanos, A. y C. Boada (2018). «Mamíferos del Ecuador. Version 2018.0.» En: ed. por J. Brito y col. Quito: Pontificia Universidad Católica del Ecuador, Museo de Zoología. Cap. Tremarctos ornatus.

Castellanos A., Altamirano M. \& Tapia G. (2005). «Ecología y comportamiento de osos andinos reintroducidos en la Reserva Biológica Maquipucuna, Ecuador: implicaciones en conservación.» En: Revista Politécnica, 54-82. Online: https: / / bit.ly /32Ft8SN, Volume = 26.

Ceballos, G. y J. Brown (1995). «Global patterns of mammalian diversity, endemism, and endangerment». En: Conservation Biology 9.3, 559-568. Online: https: / / bit. ly/2U5eWOR.

Cole, F., D. Reeder y D. Wilson (1994). «A synopsis of distribution patterns and the conservation of mammal species». En: Journal of Mammalogy 75, 266-277. Online: https:/ / bit.ly/2YWDD5d.

Cuesta, F., ed. (2000). La distribución del oso andino en el Ecuador. Quito: Fundación EcoCiencia.

Cuesta, F., M. Peralvo y D. Sánchez (2001). Métodos para investigar la disponibilidad del hábitat del oso andino: el caso de la cuenca del río Oyacachi, Ecuador. Serie Biorreserva del Cóndor 1. Quito: Fundación EcoCiencia. Online:https:/ / bit.ly/2ZYM79f.

Ferraro, P. (2002). «Global habitat protection: limitations of development interventions and a role for conservation performance payments». En: Conservation Biology 15.4, 990-1000. Online:https:/ / bit.ly/2ZiPdbO.

Goldstein, I. (2002). «Spectacled bear-cattle interactions and tree nest use in Bolivia and Venezuela». En: Ursus 13, 153-156. Online: https:/ / bit.ly/2zjyJkn.

Goldstein, I. y L. Cancino (2001). Distribución y Status del Oso Andino: una iniciativa regional de conservación a largo plazo. Mérida: Andean Bear Program-WCS Northern Andes. D. Online: http:/ / wcsfrontino.ula.ve.

Himley, M. (2009). «Nature conservation, rural livelihoods, and territorial control in Andean Ecuador.» En: Geoforum 40.5, 832-842. Online: https: / / bit.ly / 2HpVYO2.

IUCN (1996). Guidelines for re-introductions. Gland: IUCN/SSC RSG. Inf. téc. International Union for the Conservation of Nature, Reintroduction Specialist Group.

IUCN (2018). The IUCN Red List of Threatened Species. Online: https : / / www.iucnredlist.org/. International 
Union for the Conservation of Nature, Reintroduction Specialist Group.

International Association for Bear Research and Management (1999). Bear Species Descriptions. Online: https: //www.bearbiology.org/. Fairbanks.

Kattan, G. y col. (2004). «Range fragmentation of the spectacled bear Tremarctos ornatus in the northern Andes». En: Oryx 38.2, 1-10. Online: https: / / bit.ly / 2KTjxB0.

Lameda, I. y F. Del Moral (2008). «The Andean Bear in the world: view of the South American Andes. I». En: nternational Bear News 17.4, 14-15. Online: https:/ / bit. ly/2ZqVGk3.

McFarland, D. (2009). «Population Estimation, Habitat Associations and Range Expansion of Black Bears in the Upper Midwest». Tesis de mtría. Madison: University of Wisconsin. Online: https:/ / bit.ly/2H5t19U.

PUCE-q, ed. (2018). Mapa de distribución potencial del oso de anteojos. Quito: Pontificia Universidad Católica del Ecuador, Bioweb.

Peralvo, M., F. Cuesta y F. van Manen (2005). «Delineating priority habitat areas for the conservation of andean bears in northern Ecuador». En: Ursus 16.2, 222-233. Online: https://bit.ly/2zk3gOG.

Peyton, B. y col. (1998). «Status of wild Andean Bears and Policies for their Management». En: Ursus 10, 87-100. Online: https:/ / bit.ly/2ZcLIn8.

Poiani, K. y col. (1998). «Biodiversity Conservation at Multiple Scales: functional Sites, landscapes, and networks». En: BioScience 50.2, 133-146. Online:https: / / bit.ly/2NwrgXu.

Rivadeneira, C. (2008). «Study of the Andean bear (Tremarctos ornatus) as a legitimate seed disperser and elements of its diet in the region of ApolobambaBolivia». En: Ecología en Bolivia 43.1, 29-40. Online: https:/ / bit.ly/2YMp5FN.

Ruiz-García, M. (2003). «Molecular population genetic analysis of the spectacled bear (Tremarctos ornatus) in the Northern Andean Area». En: Hereditas 138, 81-93. Online: https:/ / bit.ly/2N0mK2V.
Sanderson, E. y col. (2002). «A conceptual model for conservation planning based on landscape species requirements». En: Landscape and Urban Planning 58.1, 41-56. Online: https:/ / bit.ly/33UZMQW.

Secretaría de Ambiente (2014). Programa de Conservación del Oso Andino en el Nor-occidente del Distrito Metropolitano de Quito. Inf. téc. Quito: Municipio del Distrito Metropolitano de Quito. Online: https: / / bit.ly / 2X7G7tC.

Sisk, T. y col. (1994). «Identifying extinction threats: global analyses of the distribution of biodiversity and the expansion of the human enterprise». En: Bioscience 44.9, 592-604. Online: https:/ / bit.ly/33Vi2JW.

Suárez, L. (1985). «Hábitos y distribución estacional del oso de anteojos, Tremarctos ornatus, en el páramo suroriental del volcán Antisana, Ecuador». Tesis de Grado. Quito: Pontifica Universidad Católica, Departamento de Ciencias Biológicas.

Teta, P. y col. (2018). «Lista revisada de los mamíferos de Argentina». En: Mastozoología Neotropical 25.1, 163-198. Online: https:/ / bit.ly/2PdZlxU.

Tirira (2007). Nombres de los Mamíferos del Ecuador. Publicación Especial de los Mamíferos del Ecuador 5. Quito: Ediciones Murciéélago Blanco y Museo Ecuatoriano de Ciencias Naturales.

Treves, A. y col. (2006). "Co-managing human-wildlife conflicts: a review». En: Human Dimensions of Wildlife 11.6, 383-396. Online: https:/ / bit.ly/2zmEXzS.

Troya, V. (2002). Food habits of the Andean bear in different vegetation types in the Oyacachi river basin. Quito: Fundación EcoCiencia.

WCS: Wildlife Conservation Society (2002). La selección de especies paisaje. Online: https://bit.ly/2SymCH2.

Whited, D. y col. (2000). «The importance of local and regional factors in predicting effective conservation: Planning strategies for wetland bird communities in agricultural and urban landscapes». En: Landscape and Urban Planning 49.1, 49-65. Online: https: / / bit.ly / 33X5zWf. 